\title{
Bacillus subtilis expressing dsVP28 improved shrimp survival from WSSV challenge
}

\author{
Hirun Saelim $^{\mathrm{a}, \mathrm{b}}$, Suvit Loprasert ${ }^{\mathrm{c}}$, Amornrat Phongdara ${ }^{\mathrm{a}, \mathrm{b}, *}$ \\ ${ }^{a}$ Department of Molecular Biotechnology and Bioinformatics, Faculty of Science, Prince of Songkla \\ University, Songkhla 90112 Thailand \\ b Center for Genomics and Bioinformatics Research, Faculty of Science, Prince of Songkla University, \\ Songkhla 90112 Thailand \\ c Laboratory of Biotechnology, Chulabhorn Research Institute, Bangkok 10210 Thailand
}

*Corresponding author, e-mail: pamornra@yahoo.com

Received 27 Sep 2019

Accepted 30 Jan 2020

\begin{abstract}
White spot syndrome virus (WSSV) is a potent shrimp viral pathogen responsible for significant economic losses to shrimp aquaculture all over the world. Several studies have demonstrated efficient RNAi-based approaches for suppressing viral genes and reducing shrimp mortality. However, the application has been difficult in real practical use. The present work aimed to develop an efficient approach for the delivery of VP28 specific-siRNA using Bacillus subtilis, a novel live oral vaccine vehicle, to protect shrimp against WSSV infection. A plasmid that can function in both bacteria and eukaryotic cells was developed by combining pBE-sDNA and the PCMV promoter from the pcDNA4 plasmid to obtain pBE:cDNA4. Subsequently, to evaluate the efficacy of pBE:cDNA4 in vivo, dsGFP-expressing pBE:cDNA4 (pBE:cDNA4-dsGFP) and dsVP28-expressing pBE:cDNA4 (pBE:cDNA4-dsVP28) were used to knockdown target transcripts in WSSV-infected shrimp. A high survival rate was shown for WSSV-infected shrimp injected with pBE:cDNA4-dsVP28 at 7-14 days post-infection (dpi). The pBE:cDNA4-dsVP28 plasmid was transformed into $B$. subtilis, and $B$. subtilis was used as a delivery vehicle during oral feeding. WSSV-infected shrimp fed with $B$. subtilis carrying pBE:cDNA4-dsVP28 showed $91.67 \%$ survival compared to the control, which showed only $28.57 \%$ survival. The results here demonstrate the efficient delivery of specific siRNAs by using bactofection with B. subtilis.
\end{abstract}

KEYWORDS: white spot syndrome virus, RNA interference, VP28, bactofection, B. subtilis

\section{INTRODUCTION}

White spot syndrome virus (WSSV) is the most serious cause of disease in Penaeid shrimp, generating huge economic losses. The control of viral infection is currently one of the most important challenges regarding shrimp culture [1]. Thus, applications of modern technology in shrimp farming are needed to boost production and prevent economic loss.

RNA interference (RNAi) technology is based on recent discoveries of molecular processes used by host cells to prevent the expression of foreign genetic material [2]. Major proteins involved in the RNAi pathway, including dicer and argonaute, have been identified in Penaeus monodon, confirming the existence of the RNAi mechanism in shrimp $[3,4]$. To date, RNAi is rapidly becoming a powerful tool for gene silencing applications, and these particular RNAi techniques would be very useful for developing viral vaccines to use in shrimp culture.

A number of studies performed in shrimp have demonstrated efficient RNAi-based approaches for controlling shrimp virus. Exogenous doublestranded RNAs (dsRNAs) targeting the viral gene [5] or endogenous shrimp genes essential for viral infection/replication are injected or administered into shrimp prior to viral challenge $[6,7]$. As a result, the replication of the particular virus is suppressed, significantly reducing shrimp mortality.

Among WSSV proteins, VP28 is a popular protein target for controlling WSSV spread due to its location in the viral envelope and the role in WSSV invasion into shrimp $[8,9]$. To restrict WSSV infectivity, several studies have demonstrated that injection or administration of dsRNA homologous to VP28 mRNA can induce protection against WSSV in shrimp through RNA interference $[10,11]$. To ease the use of RNAi in shrimp farming, a laboursaving, cost-effective and easy to perform siRNA delivery method has been developed. For example, 
VP28-specific siRNAs were transferred into shrimp via oral administration by using $E$. coli as a vehicle. The other method was feeding the animal with VP28dsRNA-chitosan nanoparticles $[12,13]$. However, the delivery of dsRNA by ingestion is less effective for inducing the RNAi pathway than injection. To improve the efficiency of this process, B. subtilis, a probiotic bacterium, was selected as a vehicle to transport recombinant plasmid encoding VP28-specific siRNA into shrimp.

B. subtilis is a gram-positive bacterium generally recognized as safe (GRAS) status and classified as a novel food being used as a probiotic for both human and animal consumption [14]. B. subtilis can deliver antigens and cytokines to the systemic and mucosal immune systems via mucosal routes. Therefore, its use as a vaccine delivery system using different antigens and cytokines has been widely studied $[15,16]$. Moreover, studies have developed B. subtilis into a new tool for use as a food source and as a shuttle organism to express dsRNA in RNAi experiments [17].

Here, we developed a new bacterial plasmid that can propagate in bacterial cells and transcribe a gene of interest in eukaryotic cells, namely, pBE:cDNA4. To test whether this plasmid was suitable for targeted gene expression in vitro, we constructed pBE:cDNA4 encoding GFP- or VP28specific siRNA and investigated whether these plasmids could reduce their target transcripts in vivo, which would result in the reduction of the GFP intensity or increase shrimp survival rate after WSSV infection.

For application in shrimp aquaculture, $B$. subtilis was subsequently used as the carrier for carrying pBE:cDNA4 encoding VP28-specific siRNA (pBE:cDNA4-dsVP28) against WSSV infection. The results showed that shrimp orally fed with $B$. subtilis carrying pBE:cDNA4-dsVP28 showed a higher survival rate than controls. Overall, the novel pBE:cDNA4 plasmid and B. subtilis were efficient strategic tools for practical use in shrimp aquaculture against WSSV infection.

\section{MATERIALS AND METHODS}

\section{Experimental shrimp}

Healthy Litopenaeus vannamei (bodyweight 7-10 g) were collected from a farm in Songkhla, Thailand. Shrimp have been maintained in a laboratory aquaria at a salinity of $15 \mathrm{ppt}$ for at least 1 week before use in each experiment.

\section{WSSV preparation}

Shrimp were intramuscularly injected with $100 \mu \mathrm{l}$ of various dilutions of WSSV virus in phosphatebuffered saline (PBS). The dose of WSSV used in the immunization experiments was $1.08 \times 10^{6}$ copies; this dose resulted in the death of $50 \%$ of shrimp within 5-7 days.

\section{Construction of recombinant pBE:cDNA4 plasmid}

The pBE:cDNA4 plasmid was developed by fusion of part of a eukaryotic expression cassette derived from pcDNA4 B His/Max and the replication origin of $B$. subtilis from pBE-sDNA. Both plasmids were digested by the same two restriction enzymes, MluI and ScaI, generating 2 fragments with the sizes of $4654 \mathrm{bp}$ and $3118 \mathrm{bp}$, which were from pcDNA4 B His/Max and pBE-sDNA, respectively (Fig. 1). After ligation, the fusion plasmids were transformed into E. coli Top10 and plated onto LB agar containing $100 \mu \mathrm{g} / \mathrm{ml}$ ampicillin and $10 \mu \mathrm{g} / \mathrm{ml}$ kanamycin. Subsequently, pBE:cDNA4 was transformed into the B. subtilis strain CU1065 using a two-step transformation described in the product manual for the Takara B. subtilis secretory protein expression system (Takara, USA). The selective medium for screening $B$. subtilis harbouring pBE:cDNA4 was LB agar plates containing $10 \mu \mathrm{g} / \mathrm{ml}$ of kanamycin.

\section{Construction of long hairpin-specific dsRNA-expressing pBE:cDNA4 plasmid}

A 2-step cloning strategy was developed to construct a long-strand dsRNA hairpin expression vector [18]. The plasmid map and cloning strategy are depicted in Fig. 2A. The sizes of the cloned forward and reverse VP28-specific long-hairpin dsRNA fragments are 403 bp (base number 15-417) and 510 bp (base number 15-524), respectively. These sizes almost cover the full length of VP28 (Accession number: AY422228.1, 615 bp). The forward VP28 fragment was cloned into the pGEM-T easy vector (Promega, USA) and digested by HindIII and BamHI for the subsequent cloning step into the pBE:cDNA4 vector. For the reverse VP28 fragment, the reverse sequence was cloned into the pGEM-T easy vector, which was then cut by BamHI and EcoRI to release a reverse repeat flanked by the restriction sites. Finally, the reverse repeat was subcloned into the pBE:cDNA4 plasmid containing the forward VP28 fragment using T4 DNA ligase (Promega, USA). The recombinant plasmid was transformed into $E$. coli Top10 and digested by restriction enzymes to obtain 

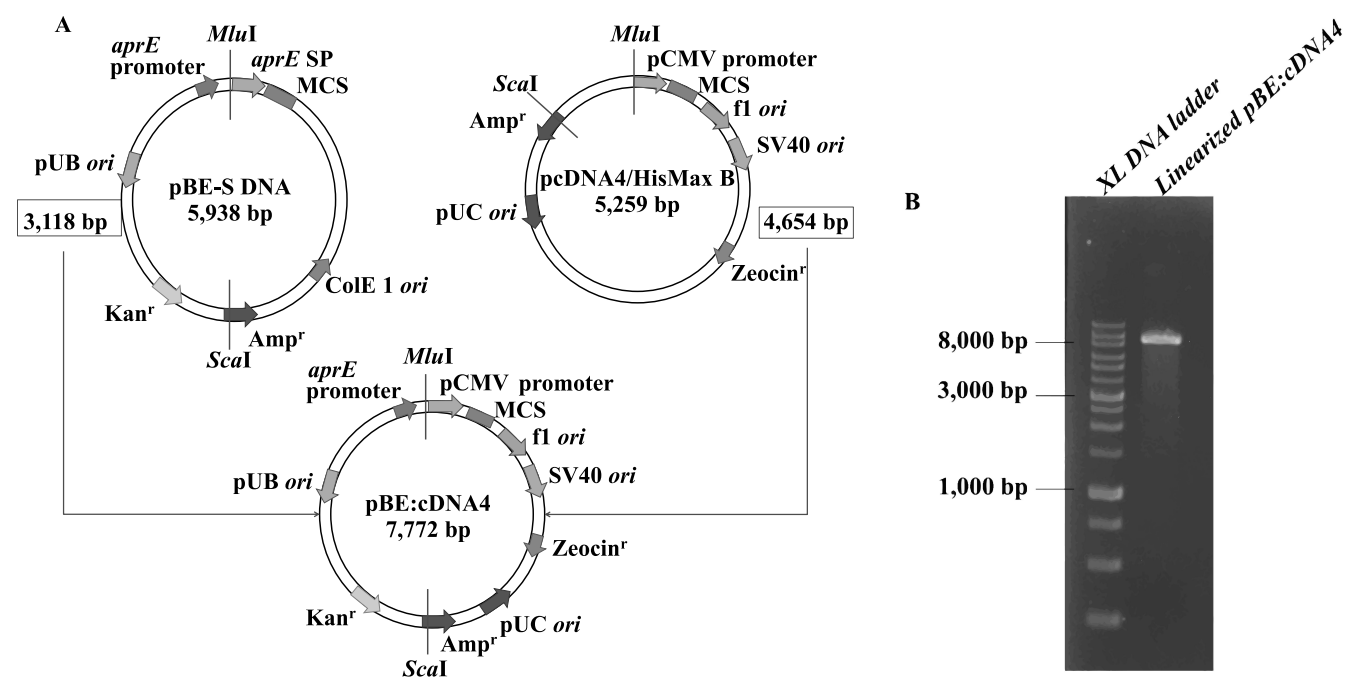

Fig. 1 Schematic representation of the pBE:cDNA4 construction strategy. (A) Ligation of MluI- and ScaI-digested pBEsDNA and pcDNA4/HisMax B plasmid products resulting in the creation of the new DNA vaccine reporter plasmid pBE:cDNA4. (B) The linearized pBE:cDNA4 shuttle plasmid (7.772 Kb).
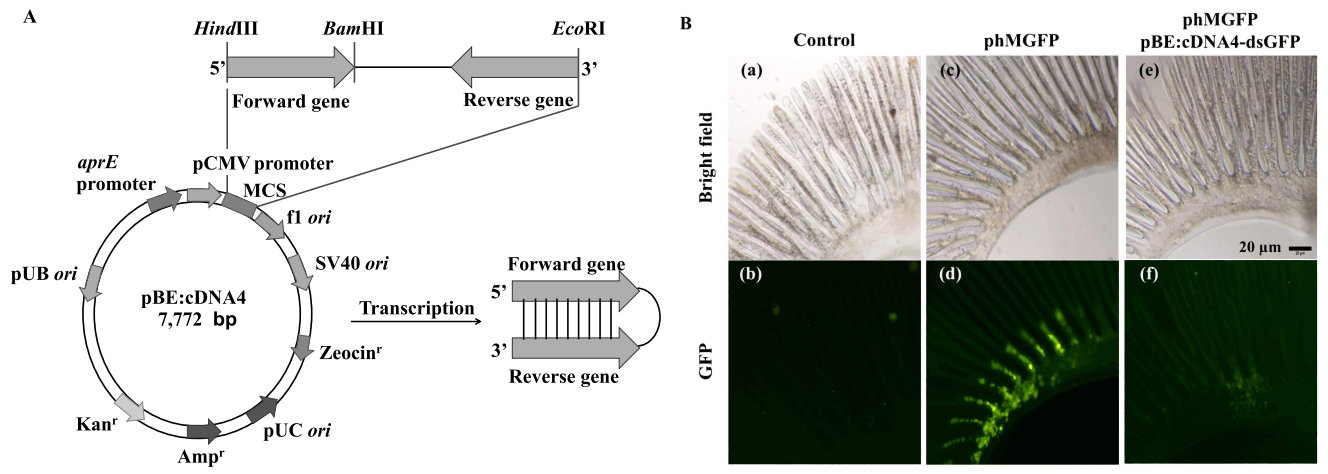

Fig. 2 Plasmid features for transcribing hairpin dsRNAs in vivo. (A) A plasmid containing the target gene configured as a forward repeat with a reverse fragment flanked by a single promoter (pCMV) can be generated in two steps. First, a forward fragment is inserted behind the promoter using the HindIII and BamHI restriction sites. Second, a reverse DNA fragment is then inserted behind the first fragment using BamHI and EcoRI restriction sites. (The HindIII, BamHI, and EcoRI restriction sites can be created using appropriately designed PCR primers.) (B) Effective inhibition of GFP production by RNAi in shrimp gills, including normal shrimp (a-b), shrimp injected with phMGFP (c-d) and shrimp injected with phMGFP and pBE:cDNA-dsGFP (e-f).

the correctly sized hairpin product. For the GFPderived hairpin, nonspecific control, the template of full-length GFP gene is 684 bp (Accession number: AY218848.1). The forward and reverse fragments are 392 bp (base number 5-396) and 583 bp (base number 5-587), respectively. The primers for constructing the VP28 and GFP plasmids are listed in Table S1. The purified pBE:cDNA4-dsVP28 and pBE:cDNA4-dsGFP plasmids were transformed into the B. subtilis strain CU1065.

\section{Evaluation of the dsGFP encoded from pBE:cDNA4-dsGFP in shrimp}

The efficacy of dsRNA expression from the pBE:cDNA4 plasmid to inhibit the target transcript was determined. In this step, exotic GFP was knocked down with GFP specific-dsRNA transcribed from pBE:cDNA4-dsGFP plasmid as a model to confirm that dsGFP could inhibit the GFP protein. Shrimp were divided into the following three groups, each group containing 3 shrimp: (1) PBS-injected shrimp (negative control), (2) phMGFP with pBE:cDNA4-dsGFP plasmid- 
injected shrimp, and (3) empty phMGFP plasmidinjected shrimp (positive control for the appearance of the green fluorescence signal), by injecting the individual shrimp with $40 \mu \mathrm{g}$ of each plasmid. The effectiveness of the GFP-specific dsRNA was monitored by measuring the intensity of GFP in shrimp gills at $48 \mathrm{~h}$ post-injection using a fluorescence microscope. To measure the GFP intensity, the shrimp gills from each group were collected, fixed with $4 \%$ paraformaldehyde for $15 \mathrm{~min}$, and then washed three times with PBS. The GFP intensity in the samples was visualized using a fluorescence microscope.

\section{Protection of shrimp by injection of pBE:cDNA-dsVP28 plasmid against WSSV infection}

Shrimp were divided into four groups with each group containing 10 shrimp. Shrimp in the first and second groups were injected with $40 \mu \mathrm{g}$ of pBE:cDNA4-dsGFP and pBE:cDNA4-dsVP8 plasmid into the third abdominal segment, respectively. The third and fourth shrimp groups were injected with PBS alone. On the 3rd day after plasmid injection, shrimp in the first, second, and third groups were challenged by WSSV. In this experiment, the third and fourth groups were used as a positive and negative control, respectively. The mortality was recorded for 14 days and the shrimp survival rate was calculated. Additionally, VP28 transcript expression was also randomly investigated from gills in both dead and surviving shrimp by RT-PCR at the end of the experiment. The VP28 specific primers are shown in Table S1. The EF- $1 \alpha$ gene was used as an internal control. The experiments were performed in triplicate.

\section{Protection of shrimp against WSSV infection by oral administration with $B$. subtilis containing pBE:cDNA4-dsRNA}

\section{Coating of feed diets}

To prepare the diet incorporating $B$. subtilis, $B$. subtilis suspensions containing pBE:cDNA4-dsVP28 or pBE:cDNA-dsGFP $\left(10^{6} \mathrm{CFU} / \mathrm{ml}\right)$ were mixed per $0.1 \mathrm{~g}$ of commercial diet. The diet incorporating B. subtilis was incubated on ice for $15 \mathrm{~min}$ to allow absorption of the bacterial suspension into the diets. The diet was pelleted by pressing through a syringe $(10 \mathrm{ml})$. PBS- and empty B. subtilis-mixed diets were used as controls. The diets were dried at room temperature for 2-3 days and stored at $4{ }^{\circ} \mathrm{C}$ until use.

\section{WSSV challenge test}

Shrimp were divided into 5 groups with each group containing 10 shrimp. Shrimp in the first and second groups were fed with $B$. subtilis containing pBE:cDNA4-dsGFP and pBE:cDNA4-dsVP28, respectively. The third group was fed with empty B. subtilis. The fourth and fifth groups were fed with PBS-mixed diets. The diets were fed for 7 days before challenging with WSSV, and feeding continued throughout the experiment. After that, shrimp in the first through fourth groups were challenged with WSSV. The mortality was recorded for 14 days and the shrimp survival rate was calculated. Additionally, VP28 transcript expression was also investigated as described above. The experiments were performed in triplicate.

\section{Statistical analysis}

Data were expressed as the mean \pm SD. The significance of the percentage survival observed in the different experimental groups was analyzed by oneway ANOVA at $95 \%$ confidence level $(p<0.05)$.

\section{RESULTS AND DISCUSSION}

\section{Construction of pBE:cDNA4}

The pBE:cDNA4 shuttle plasmid $(7.772 \mathrm{~Kb})$ was constructed using a pBE-S DNA fragment $(3.118 \mathrm{~Kb})$ that was ligated to a pcDNA4/HisMax B plasmid fragment $(4.654 \mathrm{~Kb})$. As illustrated in Fig. 1, the new plasmid harbours a eukaryotic region containing pCMV, a multiple cloning site (MCS), a T7 primer binding site for sequencing and the $\mathrm{BGH}$ polyadenylation signal polyA necessary for correct mRNA maturation. The prokaryotic region contains a pUB origin of replication, kanamycin resistance gene $\left(\operatorname{Kan}^{\mathrm{r}}\right)$ for $B$. subtilis selection, a pUC origin of replication, and an ampicillin-resistant $\left(A m p^{r}\right)$ gene for $E$. coli selection. The new plasmid was successfully stabilized in E. coli Top10 and in B. subtilis CU1065.

\section{Construction of the pBE:cDNA4-dsGFP vector and its inhibitory efficacy on exotic GFP protein in shrimp}

A 2-step cloning strategy was developed to construct a long-strand dsRNA hairpin-expression vector. The plasmid map and cloning strategy are depicted in Fig. 2A. For the VP28-specific hairpin, the sizes of the cloned forward and reverse fragments were 403 and $510 \mathrm{bp}$, respectively. For the GFP-derived hairpin nonspecific control, the forward and reverse fragments were 392 and 583 bp, respectively. 


$$
\mathbf{A}
$$

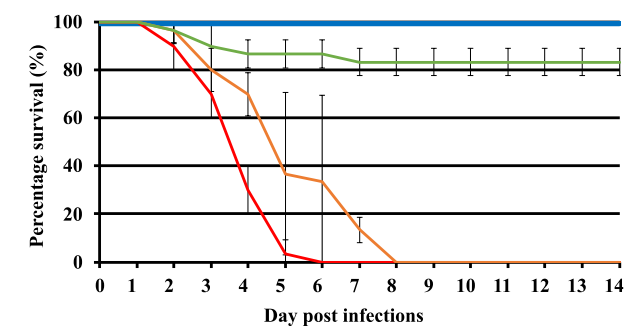

- PBS (Negative) - PBS+WSSV (Positive)

- pBE:cDNA4dsGFP+WSSV — pBE:cDNA4dsVP28+WSSV PBS PBS+WSSVPBE:cDNA4 pBE:cDNA4 (Negative) (Positive) dsGFP+WSSV dsVP28+WSSV

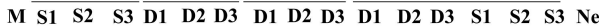

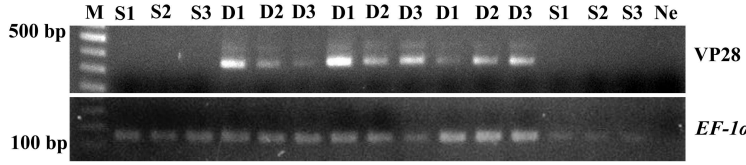

Fig. 3 Percentage survival of $L$. vannamei that was injected with PBS, pBE:cDNA4-dsGFP and pBE:cDNA4dsVP28 3 days prior to WSSV injection. (A) Shrimp injected with PBS and the PBS and WSSV mixture were used as negative and positive controls, respectively. The test groups were pBE:cDNA-dsGFP-dsVP28- and pBE:cDNAdsGFP-injected shrimp. (B) Determination of VP28 mRNA expression by RT-PCR. RNA was isolated from gill tissues of moribund (D1-D3) and surviving (S1-S3) shrimp. EF$1 \alpha$ was used as an internal control.

To evaluate the effectiveness of dsRNA expression, green fluorescent protein (GFP) expression was compared between the shrimp injected with phMGFP+pBE:cDNA4-dsGFP and phMGFP alone. GFP protein expression in shrimp gills was investigated by fluorescence microscopy at $48 \mathrm{~h}$ after injection. As shown in Fig. 2B, GFP protein expression was evident in shrimp gills after injection with phMGFP plasmid (middle). The shrimp injected with phMGFP+pBE:cDNA4-dsGFP showed lower GFP protein expression levels than the shrimp injected with phMGFP alone. GFP protein was not present in the gills of normal shrimp, which was served as a negative control. These results suggested that dsGFP against GFP was produced from pBE:cDNA4 and is capable of specifically knocking down the GFP expression in shrimp cells.

\section{Protection of shrimp by pBE:cDNA-dsVP28 immunization}

Shrimp survival in each treatment group after WSSV infection was monitored daily to examine the efficiency of antiviral immunity elicited by dsRNA corresponding to VP28 gene expressed from pBE:cDNA4. The transcription of specific dsR-

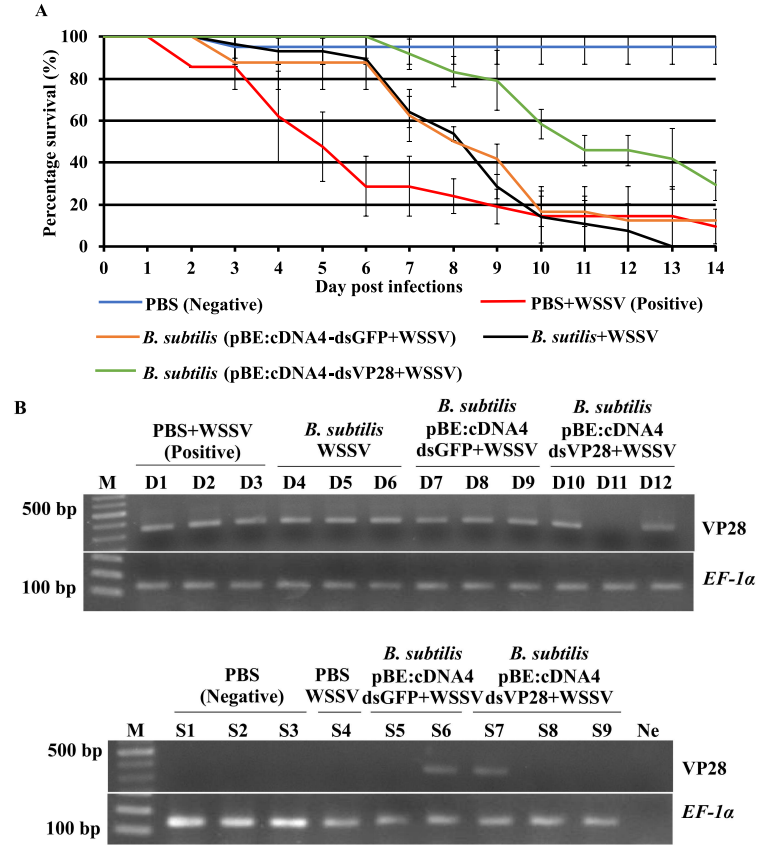

Fig. 4 Percentage of survival rate of $L$. vannamei fed with diets containing empty B. subtilis and B. subtilis carrying pBE:cDNA4 expressing either dsVP28 or dsGFP for 7 days before WSSV challenge. The shrimp survival rate was recorded every day for 14 days after WSSV challenge. (A) Determination of VP28 mRNA expression by RT-PCR from the gills of moribund (D1-12) and surviving (S1-S9) shrimp. EF-1 $\alpha$ was used as an internal control.

NAs within eukaryotic cells can be investigated by injecting shrimp with DNA constructs designed to express dsRNA. Shrimp were challenged by WSSV on the 3rd day after immunization. The groups of shrimp injected with pBE:cNDA4-dsVP8, pBE:cNDA4-dsGFP and PBS (positive control) were challenged with WSSV. In total, 83\% survival was observed in the shrimp injected with pBEcDNA4dsVP28, while $100 \%$ mortality was observed in the shrimp injected with pBEcDNA4-dsGFP and the positive control shrimp (Fig. 3A).

VP28 expression was investigated in the dead and surviving shrimp by RT-PCR to confirm WSSV infection. The survivors showed no sign of disease and VP28 expression was not detected (Fig. 3B).

\section{Delivery of pBE:cDNA4-dsVP28 expressing dsVP28 into shrimp using $B$. subtilis as a delivery vehicle}

Based on the above results, shrimp injected with pBE:cDNA4-dsVP28 were resistant to WSSV infection. In an attempt to obtain an efficient approach 
for the delivery of the antiviral dsVP28 expressed from pBE:cDNA4-dsVP28 into shrimp, B. subtilis was selected as a delivery vehicle. Shrimp were fed with food diets containing empty $B$. subtilis or B. subtilis containing pBE:cDNA4 expressing either dsVP28 or dsGFP for 7 days before WSSV challenge. After the WSSV challenge, the percentage survival of shrimp was observed for 14 days post-infection. At 7 days post-infection, shrimp in the negative control showed the highest survival rate of $95.2 \%$, and its survival rate was stable throughout the experiment. Shrimp fed with a diet containing $B$. subtilis carrying pBE:cDNA4-dsVP28 showed the highest survival rate of $91.7 \%$ while shrimp fed with a diet containing B. subtilis carrying pBE:cDNA4-dsGFP and empty B. subtilis showed survival rates of $62.5 \%$ and $64.1 \%$, respectively. The WSSV-infected shrimp fed with a commercial diet (positive control) showed the lowest survival rate of $28.6 \%$. At $14 \mathrm{dpi}$, WSSVinfected shrimp fed with a diet containing $B$. subtilis carrying pBE:cDNA4-dsVP28 had a higher survival rate than those fed with a diet containing $B$. subtilis carrying pBE:cDNA4-dsGFP and the positive control, with survival rates of $29.2 \%, 12.5 \%$ and $9.5 \%$, respectively. In contrast, no shrimp survived among the WSSV-infected shrimp fed with a diet containing empty B. subtilis (Fig. 4A). Taken together, the novel pBE:cDNA4 plasmids could be used in vivo, and bactofection of a DNA vaccine using B. subtilis was an efficient strategy for the delivery of pBE:cDNA4 expressing specific siRNAs to protect shrimp against WSSV infection. Additionally, we also investigated VP28 mRNA expression in moribund and surviving shrimp (Fig. 4B).

\section{DISCUSSION}

The RNAi pathway is thought to be an ancient mechanism for protecting the host genome against virus genetic elements through mRNA degradation induced by double-stranded RNA (dsRNA) in a sequence-specific manner $[19,20]$. Several studies have reported that siRNAs or dsRNA serves as potential therapeutic agents for treating viral disease [21, 22]. In shrimp, dsRNA is a potent trigger of an innate immune response against viruses, and dsRNA induces antiviral protection as described in L. vannamei infected with viruses including white spot syndrome virus [23], Taura syndrome virus [24], yellow head virus [25], Penaeus stylirostris densovirus [26]. The VP28 has been identified as a viral surface protein that plays a key role in the initial steps of systemic WSSV infection in shrimp [27]. Several reports have experimentally tested dsRNAs targeting the structural gene VP28 for their efficacy to confer prevention of WSSV infection in $P$. monodon and L. vannamei $[28,29]$. Thus, RNAi technology is now applicable as a prevention strategy against WSSV infection in shrimp. Recombinant VP28 protein is also effective in the prevention of WSSV infection when added to shrimp feed [30,31]. This work demonstrates a new strategy using a bacterium gene delivery system for the production of VP28 dsRNA in shrimp cells. A number of pathogens having invasive properties, such as Shigella, Salmonella, Yersinia, and Listeria, have been exploited for their use in DNA delivery to mammalian cells [32]. However, the risk of reverting to a virulent phenotype cannot be completely mitigated, and there remains a need to search for safer alternatives $[33,34]$. B. subtilis was exploited as a safer alternative compared with invasive pathogens. Several studies have demonstrated that oral administration of $B$. subtilis expressing VP28 protein can induce protection in shrimp against WSSV [35-37].

In the present study, noninvasive $B$. subtilis was explored as a DNA carrier for the delivery of a newly constructed plasmid, pBE:cDNA4 $(7.772 \mathrm{~Kb}$ in size) Fig. 1, to eukaryotic cells. The pBE:cDNA4 offers several attractive features as a DNA vaccine candidate, especially as it is capable of replicating in both prokaryotic cells such as $E$. coli and $B$. subtilis and eukaryotic cells. We evaluated the efficacy of dsGFP expressed from the pBE:cDNA4dsGFP vector to interfere with green fluorescent protein (GFP) transgene expression. The results showed that the GFP intensity in gills of pBE:cDNA4dsGFP-injected shrimp was decreased (Fig. 2B). This implied that dsRNA could be produced in-frame from the pBE:cDNA4 plasmid and was capable of effective gene-specific knockdown of target gene expression in shrimp. The pBE:cDNA4-dsVP28 expressing dsVP28 targeting WSSV VP28 was also constructed. The shrimp survival rate in WSSVinfected shrimp injected with pBE:cDNA4-dsVP28 was higher than in WSSV-infected shrimp injected with pBE:cDNA4-dsGFP (Fig. 3A). After checking the VP28 transcript, the results showed a reduction in VP28 transcriptional levels in WSSV-infected shrimp injected with pBE:cDNA4-dsVP28 compared to WSSV-infected shrimp injected with pBE:cNDA4dsGFP and the control group, indicating that the protective effects against WSSV infection depended on dsRNA specific to VP28 expression in shrimp. It is clear that VP28-specific dsRNA expression regulated by the pCMV promoter in pBE:cDNA4 promoted protective effects against WSSV infection. 
To promote the use of RNAi in shrimp farming, $B$. subtilis had been used as a delivery vehicle. Recombinant B. subtilis was developed by transformation with pBE:cDNA4-dsGFP. After oral administration of recombinant $B$. subtilis to WSSVinfected shrimp, the highest survival rate was shown for WSSV-infected shrimp fed with a diet containing B. subtilis carrying the pBE:cDNA4-dsVP28 plasmid. Moreover, VP28 mRNA in WSSV-infected shrimp fed with a diet containing $B$. subtilis carrying the pBE:cDNA4-dsVP28 plasmid was not present in surviving shrimp. This revealed that oral delivery of the plasmid expressing dsVP28 could activate the RNAi pathway, drastically suppressing VP28 mRNA expression.

The B. subtilis bactofection mechanism in shrimp could be similar to the Lactococcus lactis, a gram-positive lactic acid bacterium, bactofection mechanism in mice [38]. B. subtilis probably adheres to epithelial cells and enters the cell by internalization of a vacuole/phagosome. After vacuole/phagosome lysis, plasmid DNA was released and translocated into the nucleus, resulting in the expression of the gene of interest $[39,40]$. This dsRNA delivery system may overcome several limitations associated with the biomolecule application to prevent and protect shrimp from acquiring diseases during aquaculture.

\section{Appendix A. Supplementary data}

Supplementary data associated with this article can be found at http://dx.doi.org/10.2306/ scienceasia1513-1874.2020.S003.

Acknowledgements: This work is supported by the Royal Golden Jubilee Graduate Program from the Thailand Research Fund (TRF) (Grant No. PHD/0253/2553) to Hirun Saelim (4.J.PS/53/A.1) and the government budget of Prince of Songkla University (Grant No. SCI620073S). The authors would like to thank Ponsit Sathapondecha for the experimental design of siRNA.

\section{REFERENCES}

1. Powell D, Knibb W, Nguyen NH, Elizur A (2016) Transcriptional profiling of banana shrimp Fenneropenaeus merguiensis with differing levels of viral load. Integr Comp Biol 56, 1131-1143.

2. Reshi ML, Wu JL, Wang HV, Hong JR (2014) RNA interference technology used for the study of aquatic virus infections. Fish Shellfish Immun 40, 14-23.

3. Unajak S, Boonsaeng V, Jitrapakdee S (2006) Isolation and characterization of cDNA encoding Argonaute, a component of RNA silencing in shrimp (Penaeus monodon). Comp Biochem Physiol B Biochem Mol Biol 145, 179-187.
4. Su J, Oanh DT, Lyons RE, Leeton L, Van Hulten MC, Tan SH, Song L, Rajendran K, et al (2008) A key gene of the RNA interference pathway in the black tiger shrimp, Penaeus monodon: identification and functional characterisation of Dicer-1. Fish Shellfish Immun 24, 223-233.

5. Chimwai C, Tongboonsong P, Namramoon O, Panyim S, Attasart P (2016) A formulated double-stranded RNA diet for reducing Penaeus monodon densovirus infection in black tiger shrimp. J Invertebr Pathol 134, 23-26.

6. Attasart P, Namramoon O, Kongphom U, Chimwai C, Panyim S (2013) Ingestion of bacteria expressing dsRNA triggers specific RNA silencing in shrimp. Virus Res 171, 252-256.

7. Sanitt P, Attasart P, Panyim S (2014) Protection of yellow head virus infection in shrimp by feeding of bacteria expressing dsRNAs. J Biotechnol 179, 26-31.

8. Zhang X, Huang C, Xu X, Hew CL (2002) Identification and localization of a prawn white spot syndrome virus gene that encodes an envelope protein. $J$ Gen Virol 83, 1069-1074.

9. Wu W, Wang L, Zhang X (2005) Identification of white spot syndrome virus (WSSV) envelope proteins involved in shrimp infection. Virology 332, 578-583.

10. Liu C, Li F, Sun Y, Zhang X, Yuan J, Yang H, Xiang $\mathrm{J}$ (2016) Virus-derived small RNAs in the penaeid shrimp Fenneropenaeus chinensis during acute infection of the DNA virus WSSV. Sci Rep 6, ID 28678.

11. Nilsen P, Karlsen M, Sritunyalucksana K, Thitamadee S (2017) White spot syndrome virus VP28 specific double-stranded RNA provides protection through a highly focused siRNA population. Sci Rep 7, ID 1028.

12. Thammasorn T, Sangsuriya P, Meemetta W, Senapin S, Jitrakorn S, Rattanarojpong T, Saksmerprome V (2015) Large-scale production and antiviral efficacy of multi-target double-stranded RNA for the prevention of white spot syndrome virus (WSSV) in shrimp. BMC Biotechnol 15, ID 110.

13. Sarathi M, Simon MC, Venkatesan C, Hameed AS (2008) Oral administration of bacterially expressed VP28dsRNA to protect Penaeus monodon from white spot syndrome virus. Mar Biotechnol 10, 242-249.

14. Rosales-Mendoza S, Angulo C, Meza B (2016) Foodgrade organisms as vaccine biofactories and oral delivery vehicles. Trends Biotechnol 34, 124-136.

15. Tang Z, Sun H, Chen T, Lin Z, Jiang H, Zhou X, Shi C, Pan H, et al (2017) Oral delivery of Bacillus subtilis spores expressing cysteine protease of Clonorchis sinensis to grass carp (Ctenopharyngodon idellus): induces immune responses and has no damage on liver and intestine function. Fish Shellfish Immun 64, 287-296.

16. Jiang H, Bian Q, Zeng W, Ren P, Sun H, Lin Z, Tang Z, Zhou X, et al (2019) Oral delivery of Bacillus subtilis spores expressing grass carp reovirus VP4 protein 
produces protection against grass carp reovirus infection. Fish Shellfish Immun 84, 768-780.

17. Lezzerini $M$, van de Ven $K$, Veerman $M$, Brul S, Budovskaya YV (2015) Specific RNA interference in Caenorhabditis elegans by ingested dsRNA expressed in Bacillus subtilis. PLoS One 10, ID e0124508.

18. Saksmerprome V, Charoonnart P, Gangnonngiw W, Withyachumnarnkul B (2009) A novel and inexpensive application of RNAi technology to protect shrimp from viral disease. $J$ Virol Methods 162, 213-217.

19. Zamore PD, Tuschl T, Sharp PA, Bartel DP (2000) RNAi: double-stranded RNA directs the ATPdependent cleavage of mRNA at 21 to 23 nucleotide intervals. Cell 101, 25-33.

20. Zhou R, Rana TM (2013) RNA-based mechanisms regulating host-virus interactions. Immunol Rev 253, 97-111.

21. Ma Y, Chan CY, He ML (2007) RNA interference and antiviral therapy. World $J$ Gastroenterol 13, 5169-5179.

22. Robalino J, Browdy CL, Prior S, Metz A, Parnell P, Gross P, Warr G (2004) Induction of antiviral immunity by double-stranded RNA in a marine invertebrate. $J$ Virol 78, 10442-10448.

23. Mejía-Ruíz $\mathrm{CH}$, Vega-Peña $\mathrm{S}$, Alvarez-Ruiz P, Escobedo-Bonilla CM (2011) Double-stranded RNA against white spot syndrome virus (WSSV) vp28 or vp26 reduced susceptibility of Litopenaeus vannamei to WSSV, and survivors exhibited decreased susceptibility in subsequent re-infections. $J$ Invertebr Pathol 107, 65-68.

24. Ongvarrasopone C, Saejia P, Chanasakulniyom M, Panyim S (2011) Inhibition of Taura syndrome virus replication in Litopenaeus vannamei through silencing the LvRab7 gene using double-stranded RNA. Arch Virol 156, 1117-1123.

25. Sanitt P, Apiratikul N, Niyomtham N, Yingyongnarongkul BE, Assavalapsakul W, Panyim S, Udomkit A (2016) Cholesterol-based cationic liposome increases dsRNA protection of yellow head virus infection in Penaeus vannamei. J Biotechnol 228, 95-102.

26. Ho T, Yasri P, Panyim S, Udomkit A (2011) Doublestranded RNA confers both preventive and therapeutic effects against Penaeus stylirostris densovirus (PstDNV) in Litopenaeus vannamei. Virus Res 155, 131-136.

27. Van Hulten MC, Witteveldt J, Snippe M, Vlak JM (2001) White spot syndrome virus envelope protein VP28 is involved in the systemic infection of shrimp. Virology 285, 228-233.

28. Jariyapong P, Weerachatyanukul W, Direkbusarakom S, Hirono I, Wuthisuthimethavee S, Chotwiwatthanakun C (2015) Enhancement of shrimp immunity against white spot syndrome virus by Macrobrachium rosenbergii nodavirus-like particle encapsulated VP28 double-stranded RNA. Aquaculture 446, 325-332.

29. Jariyapong P, Chotwiwatthanakun C, Pooljun C, Weerachatyanukul W (2019) Infectious hypodermal and hematopoietic necrosis virus-like particles encapsulating VP28 double-stranded RNA protect shrimp from white spot syndrome virus. Aquaculture 504, 260-266.

30. Kwang J (2011) Oral vaccination of baculovirusexpressed VP28 displays enhanced protection against white spot syndrome virus in Penaeus monodon. PLoS One 6, ID e26428.

31. Musthaq SKS, Kwang J (2014) Evolution of specific immunity in shrimp - A vaccination perspective against white spot syndrome virus. Dev Comp Immunol 46, 279-290.

32. Schoen C, Stritzker J, Goebel W, Pilgrim S (2004) Bacteria as DNA vaccine carriers for genetic immunization. Int $J$ Med Microbiol 294, 319-335.

33. Medina E, Guzmán CA (2001) Use of live bacterial vaccine vectors for antigen delivery: potential and limitations. Vaccine 19, 1573-1580.

34. Bahey-El-Din M (2012) Lactococcus lactis-based vaccines from laboratory bench to human use: an overview. Vaccine 30, 685-690.

35. Fu LL, Shuai JB, Xu ZR, Li JR, Li WF (2010) Immune responses of Fenneropenaeus chinensis against white spot syndrome virus after oral delivery of VP28 using Bacillus subtilis as vehicles. Fish Shellfish Immun 28, 49-55.

36. Ning D, Leng X, Li Q, Xu W (2011) Surface-displayed VP28 on Bacillus subtilis spores induce protection against white spot syndrome virus in crayfish by oral administration. J Appl Microbiol 111, 1327-1336.

37. Nguyen AT, Pham CK, Pham HT, Pham HL, Nguyen AH, Dang LT, Huynh HA, Cutting SM, et al (2014) Bacillus subtilis spores expressing the VP28 antigen: a potential oral treatment to protect Litopenaeus vannamei against white spot syndrome. FEMS Microbiol Lett 358, 202-208.

38. Mancha-Agresti P, Drumond MM, Do Carmo FLR, Santos MM, dos Santos JSC, Venanzi F, Chatel JM, Leclercq SY, et al (2017) A new broad range plasmid for DNA delivery in eukaryotic cells using lactic acid bacteria: in vitro and in vivo assays. Mol Ther Methods Clin Dev 4, 83-91.

39. De Azevedo M, Meijerink M, Taverne N, Pereira VB, LeBlanc JG, Azevedo V, Chatel JM (2015) Recombinant invasive Lactococcus lactis can transfer DNA vaccines either directly to dendritic cells or across an epithelial cell monolayer. Vaccine 33, 4807-4812.

40. Yagnik B, Padh H, Desai P (2016) Construction of a new shuttle vector for DNA delivery into mammalian cells using non-invasive Lactococcus lactis. Microbes Infect 18, 237-244. 


\section{Appendix A. Supplementary data}

Table S1 Bacterial strains, plasmids, and primers used in this study.

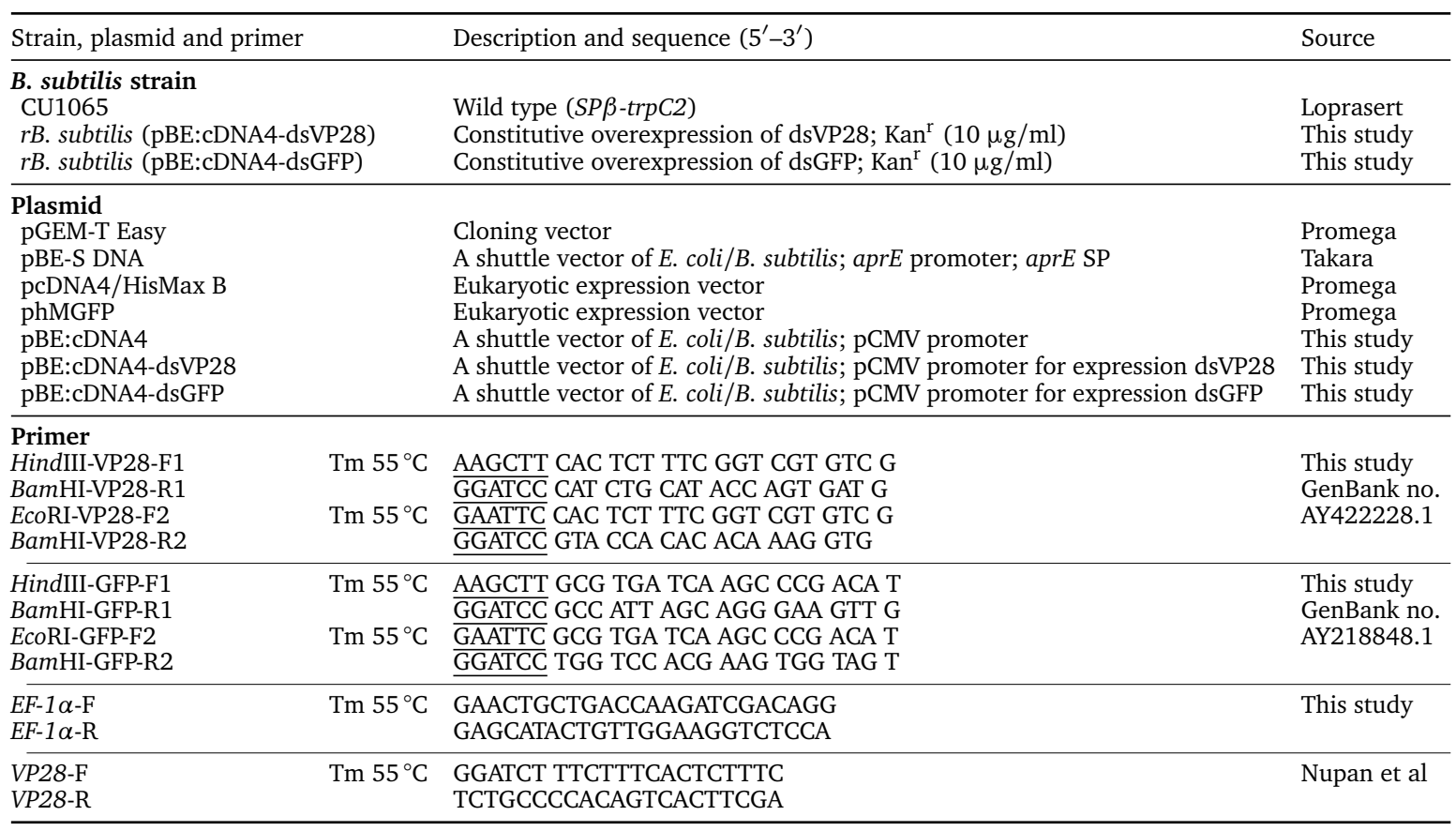

